\title{
UMA PROPOSTA DE FÓRUM INTELIGENTE PARA A PLATAFORMA MOODLE
}

\author{
Priscilla Batista Mendes, Josiane Lemos Machiavelli e Cristine Martins Gomes de Gusmão \\ Grupo SABER Tecnologias Educacionais e Sociais, Universidade Federal de Pernambuco \\ Corpo Discente da UFPE, Espaço SABER, Av. dos Economistas, s/n, Cidade Universitária, \\ Recife, PE, CEP 50.740-590, Brasil
}

\begin{abstract}
RESUMO
Fóruns de discussão são ferramentas utilizadas em Ambientes Virtuais de Aprendizagem (AVAs) como um espaço de interação entre seus participantes. No entanto, a literatura mostra que a insuficiência neste nível de interação é um problema frequente, e especialmente relevante para cursos autoinstrucionais, onde não há tutor, moderador ou professor que faça este incentivo. Assim, apresentamos um plug-in de fórum para AVAs implementado na plataforma Moodle, com características que buscam incentivar os usuários a um maior envolvimento com os cursos e melhorar sua experiência de aprendizagem. Suas principais funcionalidades são o uso da técnica de Inteligência Artificial de filtragem baseada em conteúdo, para recomendar usuários e recursos didáticos; mecanismo de curtida e não curtida de postagens, inspirado nas redes sociais; exibição de um ranking de usuários, de acordo com sua quantidade de interações no fórum; relatórios que mostram aos gestores os temas mais recorrentes nas discussões e os usuários que se destacam nas mesmas, distribuídos geograficamente, a fim de auxiliá-los na melhoria continua das ofertas educacionais. Entre os resultados obtidos com o uso desta ferramenta, destacam-se: possibilidade de atender necessidades individualizadas apresentadas pelos cursistas, a partir da indicação de recursos complementares ao aprendizado e pessoas que tenham interesse de discutir temas semelhantes; facilidade que a equipe de gestão dos cursos tem tido para conhecer as demandas dos cursistas a partir do monitoramento frequente dos relatórios que o plug-in oferece, e possibilidade de disponibilizar conteúdos e materiais didáticos complementares, a fim de apoiar os estudantes no processo de aprendizado, considerando-se, inclusive, necessidades regionalizadas, algo que não é simples de se fazer no fórum convencional do Moodle, principalmente em ofertas massivas, que envolvem milhares de usuários simultaneamente.
\end{abstract}

\section{PALAVRAS-CHAVE}

Fórum de Discussão, Cursos Autoinstrucionais Online, Inteligência Artificial, Moodle

\section{INTRODUÇÃO}

As tecnologias digitais têm tido presença cada vez mais disseminada no mundo contemporâneo, transformando todos os segmentos da sociedade, incluindo as atividades educativas. Neste contexto, emergiram os Ambientes Virtuais de Aprendizagem (AVAs), que se constituem em espaços intangíveis no qual estudantes e formadores podem se reunir e compartilhar conhecimentos, visando sua assimilação e construção conjunta. Eles se configuram como sistemas computacionais, conectados via Internet, os quais permitem a interação dos usuários, mesmo em espaço e tempos distintos (Kanuka, 2005; Loncar, Barrett e Liu, 2014). Nestes ambientes, o conteúdo, de uma maneira geral, é organizado em formato de cursos, cuja realização pode contar com a orientação de tutores, facilitadores e professores, ou não. Neste último caso, o estudante deve ter recursos que o apoiem na navegação entre as atividades, para que ele seja capaz de realizá-las por si mesmo. Também há a possibilidade de realizar atividades para consolidar a aprendizagem e avaliar a aquisição de conhecimento, as quais podem ser realizadas individualmente ou em grupo, a depender dos objetivos de aprendizagem estabelecidos pelos criadores do curso.

As atividades colaborativas têm sido reconhecidas pela literatura acadêmica como um meio bastante eficiente de aprendizagem, uma vez que a socialização do conteúdo permite que os cursistas possam complementar os seus conhecimentos mutuamente, e elevem o seu domínio dos assuntos discutidos. Em AVAs, notadamente, os fóruns de discussão têm sido utilizados com essa finalidade, constituindo-se em um dos principais meios para que os usuários possam se engajar em uma conversação tanto sobre conteúdo quanto para estabelecer laços 
sociais, o que é necessário mesmo na ausência de contato físico (Kanuka, 2005; Loncar, Barrett e Liu, 2014; Monahan, Mcardle e Bertolotto, 2008; Silva, 2008; Thomas, 2002). Apesar desses benefícios, é comum a subutilização dos fóruns. Muitas vezes, os usuários não se envolvem nas conversas ou interagem apenas para cumprir os requisitos mínimos quando a participação é obrigatória, reduzindo a eficácia da ferramenta. Desta forma, estratégias que possibilitem melhor monitoramento da participação nos fóruns, bem como estimulem a interação entre os cursistas podem ser capazes de potencializar o uso desta ferramenta (Mendes, Machiavelli e Gusmão, 2019b).

Considerando a importância das atividades colaborativas no processo de aprendizagem, este artigo apresenta uma ferramenta inovadora de fórum customizado para ambientes virtuais de aprendizagem baseados na plataforma Moodle, que faz uso da Inteligência Artificial (IA) a fim de estimular a participação dos cursistas e melhor atender suas necessidades de aprendizagem. O artigo possui a seguinte estrutura: na próxima seção, será apresentado um referencial teórico com conceitos importantes para o entendimento e concepção da proposta. Em seguida, será feita uma descrição detalhada do plug-in de fórum desenvolvido e, por fím, serão descritas as conclusões.

\section{REFERENCIAL TEÓRICO}

\subsection{Fóruns de Discussão Online}

Este tipo de fórum pode ser definido como um espaço onde os participantes podem estabelecer comunicação virtual, principalmente no formato de mensagens de texto (Kanuka, 2005), de forma horizontalizada (Barros, Santos e Romero, 2019). Isto permite a interação e o compartilhamento de ideias (Premagowrie, Vaani e Ho, 2014), favorecendo o ensino online (Mokoena, 2013), de maneira formal, como uma das atividades requeridas para a conclusão do curso, ou, simplesmente, para se engajar em uma conversa com outros integrantes da experiência de aprendizagem (Futterleib, Santos, 2006), fortalecendo os laços sociais. Estas interações fomentam a construção de comunidades virtuais, viabilizadas pela Internet, tendo como característica a flexibilidade de uso do tempo e do espaço, mas também podem ser utilizadas para complementação de atividades presenciais (Loncar, Barrett e Liu, 2014).

A inclusão de espaços de discussão permite uma experiência de aprendizagem mais aprofundada e de maior qualidade (Kanuka, 2005; Kolb, 1984), visto que os participantes podem superar suas lacunas de conhecimento com o auxílio dos demais, e podem consolidar seu aprendizado ao engajar-se nas discussões e contribuir com a aprendizagem de seus colegas. Uma consequência disto é o aumento das notas dos estudantes, como foi reportado na literatura por Webb et al. (2004). Outra vantagem desta ferramenta é a promoção do pensamento crítico e da reflexão (Kanuka, 2005; Thomas, 2002, McNamara, Brown, 2008), uma vez que neste espaço há exposição a pontos de vista diversos (Shaw, 2012), uma contribuição extremamente valiosa nesta era da informação. Dadas estas características, torna-se claro o papel dos fóruns online como meio de construção social do conhecimento (Thomas, 2002; Silva, 2008; Monahan, Mcardle e Bertolotto, 2008), disponibilizando-o a todos os sujeitos dispostos a participar deste processo.

A eficácia do fórum depende do engajamento dos estudantes e da necessidade de regularidade de participação na discussão coletiva (Kolb, 1984; Mason, 2011; Mendes, Machiavelli e Gusmão, 2019b). Webb et al., 2004, detectaram uma proporcionalidade entre o incremento das notas em um curso e a participação nos fóruns, o que significa que quanto mais os estudantes se engajam nesta atividade, melhor pode ser seu desempenho. Este achado também foi confirmado por Alstete e Beutell (2004), com a conclusão de que o indicador mais forte do desempenho dos estudantes era seu nível de participação nas discussões.

\subsection{Ambientes Virtuais de Aprendizagem}

O advento da Internet e da World Wide Web permitiu a criação de um espaço alternativo - o ciberespaço - que pode servir de extensão ou continuação dos ambientes físicos, nas mais diversas atividades humanas (Kalay, 2004). Desta forma, a partir de meados dos anos 90, surgiram produtos de software educacionais, denominados AVAs, visando dar suporte a atividades de ensino-aprendizagem online (O'Leary e Ramsden, 2002). Tais ambientes têm assumido um papel crucial em cenários de Educação a Distância (Krämer, 2002), constituindo-se em uma alternativa muito mais poderosa e abrangente que outros meios previamente utilizados 
nesta modalidade. São inúmeras as possibilidades ofertadas por esta tecnologia, incluindo: os conteúdos podem ser apresentados em diferentes formatos de mídia, tais como textos, imagens, sons, simulações, etc.; os diferentes recursos podem ser combinados de forma a possibilitar a apresentação de conteúdos complexos, com diversas representações possíveis; o usuário pode ter flexibilidade de navegação e busca das atividades e recursos disponibilizados, de acordo com seu estilo de aprendizagem e interesses; a trajetória do estudante é registrada dentro do ambiente, o que permite sua avaliação e rastreabilidade (Krämer, 2002).

Além de materiais didáticos, os AVAs podem dispor de atividades, que permitem que os estudantes pensem por si mesmos, apliquem o conhecimento, monitorem seu progresso e avaliem seu entendimento (Lockwood, 1992), ações que os auxiliam na consolidação da aprendizagem. As atividades podem ser síncronas ou assíncronas, sendo estas mais flexíveis para os usuários. Entre elas, incluem-se (Bri et al., 2009): questionários, envio de tarefas, chat, videoconferência, documentos de edição compartilhada, fóruns, blogs, etc., cuja seleção pode ser adequada de acordo com os objetivos pedagógicos dos cursos.

Existem diversos sistemas em que estes ambientes podem ser implementadas, entre opções de uso gratuito e comercial (Bri et al., 2009). Algumas das plataformas conhecidas são ATutor, WebCT, Blackboard, Desire2Learn, Sakai Project, Canvas, mas a que ocupa um lugar de destaque é a plataforma Moodle, um sistema disseminado em instituições de ensino em todo o mundo, contando com dezenas de milhares de implementações, e mais de 90 milhões de usuários. Sua popularidade deve-se à sua característica de código aberto, sob a licença GNU - General Public License, o que permite sua livre utilização, adaptação, extensão e modificação, para fins comerciais e não-comerciais (Moodle, 2018).

\subsection{Cursos Autoinstrucionais Online}

Este modelo de curso é entregue por meio de materiais autoexplicativos e objetivos, de modo que o próprio aluno tenha condições de se guiar dentro da oferta educacional, de acordo com sua disponibilidade e necessidade, exercendo sua autonomia e independência (Brown, Ceretta e Soratto, 2018), e assumindo uma maior responsabilidade quanto a seus resultados (Mengistu, 2016). Esta abordagem tem ganhado popularidade nos últimos anos (Brown, Ceretta e Soratto, 2018), especialmente entre as universidades, que têm se dedicado a prover cursos autoinstrucionais, valendo-se das tecnologias digitais de informação e comunicação, para dar a seus alunos um maior controle de tempo e espaço de aprendizagem (Krämer e Schulte, 2008).

Mengistu (2016) destaca a importância do desenvolvimento dos recursos autoinstrucionais para o sucesso da educação a distância, uma vez que estes devem assimilar funções que um professor assumiria no contexto presencial, como guiar, motivar, explicar, provocar, questionar, discutir alternativas, incentivar os estudantes em seu progresso, auxiliar, entre outras. Mas de acordo com Menon et al. (2016), se bem-feito, o aprendizado proporcionado por um curso autoinstrucional pode ser comparável à instrução recebida por um bom professor, em sala de aula. Adicionalmente, considerando que o design dos materiais didáticos exerce influência sobre o nível de engajamento dos estudantes no curso (Krämer e Schulte, 2008), cabe aos desenvolvedores dos cursos entenderem bem a natureza do conteúdo e de seu público para que ofereçam recursos adequados aos objetivos de aprendizagem pretendidos e expectativas, sendo possível, assim, obter bons resultados.

\subsection{Inteligência Artificial}

A Inteligência Artificial (IA) trata da possibilidade de que o aprendizado, ou qualquer outra característica da inteligência, pode ser descrito tão precisamente que viabiliza sua simulação por máquinas (McCarthy e Minsky, 2006). Esta abordagem apresenta grande valor, uma vez que permite desenvolver e replicar o processo de tomada de decisão de humanos (Bajaj e Sharma, 2018), ampliando a capacidade computacional, que até seu surgimento dependia de instruções explicitamente programadas, para um procedimento mais poderoso e adaptativo.

$\mathrm{Na}$ área da Educação, o potencial da IA foi logo identificado, datando do início dos anos 70 (Nwana, 1990). As suas aplicações envolvem diversas ferramentas e tecnologias, como robôs instrutores, sistemas tutores inteligentes, sistemas adaptativos, etc., e abrangem diferentes etapas do processo educacional, incluindo o desenvolvimento de conteúdos, métodos de ensino, avaliação de estudantes, e comunicação entre os participantes das ofertas educacionais (Chassignol et al., 2018). A recomendação de conteúdo, que é uma das funcionalidades abordadas neste artigo, faz referência a este último item, sendo um recurso facilitador da interação dos aprendizes com os conteúdos e seus pares, utilizando-se da IA para poder gerar sugestões de forma adaptativa e personalizada. Segundo Herath e Jayarathne (2018), os principais paradigmas para a recomendação 
de conteúdo são a filtragem colaborativa, em que são recomendados itens considerando as preferências de usuários similares a um dado usuário, e a filtragem baseada em conteúdo, que recomenda itens semelhantes àqueles que foram selecionados previamente pelo usuário. Também é possível combinar estas abordagens entre si, ou com outras técnicas computacionais, para uma melhor precisão.

\section{FÓRUM INTELIGENTE - PLUG-IN PARA A PLATAFORMA MOODLE}

\subsection{Motivação}

O plug-in de fórum aqui apresentado foi desenvolvido no contexto do Grupo de Pesquisa SABER Tecnologias Educacionais e Sociais, ligado à Universidade Federal de Pernambuco, no qual uma das principais atividades é a oferta de cursos online, implementados com a plataforma Moodle. No geral, são oferecidos dois tipos de cursos: especializações, com uma quantidade limitada de usuários por turma, advindos de um processo de seleção; e cursos autoinstrucionais, para formação continuada, em que a inscrição dos usuários é feita pelos próprios interessados, e divulgada de maneira ampla, sem a imposição de restrições. Uma distinção importante é que nos cursos de especialização os estudantes contam com o acompanhamento de tutores, o que se faz necessário dada a maior complexidade dos conteúdos, e por ser uma formação de nível superior. Enquanto isso, os autoinstrucionais, conforme sua própria definição, partem do pressuposto da autonomia do aluno em sua realização, ficando este responsável pela sua trajetória dentro do curso sem ter a figura de um tutor, facilitador ou professor que o guie. Porém, o design é pensado para que a utilização do ambiente seja feita de forma intuitiva. Além disso, os estudantes podem interagir com outros participantes por meio dos fóruns, que são idealizados como espaços de debates sobre as temáticas abordadas nos cursos.

No entanto, apesar de o recurso de fórum propiciar esta possibilidade de aprendizagem colaborativa, identificamos, de maneira recorrente, sua subutilização. Quando este problema ocorre nos cursos com tutoria, a estratégia de solução é a atuação do tutor no sentido de estimular os alunos à participação, entrando em contato com os ausentes e também provocando a ampliação na discussão. Porém, isto não é aplicável aos cursos autoinstrucionais, visto que se considerarmos a média que adotamos de 40 alunos por tutor, replicar isto nos cursos abertos, que possuem da ordem de dezenas de milhares de alunos (em nosso programa de cursos mais recente, Programa de Qualificação Profissional em Urgência e Emergência na Atenção Básica, que conta com 6 cursos, a média de usuários por curso é de quase 35.000), o que torna inviável economicamente a contratação de profissionais para realizar este acompanhamento. Por isso, sentiu-se a necessidade de criar um mecanismo automatizado de estímulo à participação, de forma que as próprias características do fórum fossem atrativas e motivassem a participação dos usuários. Inicialmente, cogitou-se adaptar a ferramenta nativa do Moodle, no entanto, foi avaliado que seria melhor desenvolver um recurso novo, seguindo o padrão de plug-in da plataforma, pois poderíamos desenvolver as funcionalidades desejadas de início, em vez de tentar adaptá-las a um código que tinha sua lógica própria e talvez não tivesse uma interação adequada com o que fosse agregado posteriormente. Assim, foi reunida uma equipe para se encarregar da execução deste projeto, composta por profissionais de tecnologia da informação, designers gráficos, profissionais da educação e gestores.

\subsection{Processo de Desenvolvimento}

O desenvolvimento da solução acompanhou as seguintes etapas: 1) Brainstorming inicial: envolveu a identificação dos requisitos, preferências e restrições no desenvolvimento do plug-in;2) Análise de viabilidade: foram feitas estimativas de recursos necessários para o desenvolvimento da ferramenta; 3) Determinação de cronograma: analisou-se o tempo estimado, dada a disponibilidade da equipe, para desenvolvimento das atividades, restrito ao calendário dos cursos em que se pretendia utilizar a ferramenta; 4) Criação de wireframe: foi feito com o objetivo de permitir a visualização da aparência inicial do software, estabelecendo uma base para o projeto gráfico definitivo; 5) Implementação do plug-in: definiu-se o uso da ferramenta de controle de versão GitLab, para auxiliar na gestão do processo de desenvolvimento, uma vez que ela permite o registro da evolução do código e sua rastreabilidade. Como o plug-in seria adicionado a um sistema previamente existente, isso simplificou a tomada de decisão das tecnologias adotadas, aderindo-se às linguagens utilizadas pela plataforma Moodle; 6) Testagem: uma vez desenvolvida a solução, procedeu-se a uma etapa de testes, realizada inicialmente por dois desenvolvedores. Após a correção dos erros identificados por eles, passou-se a uma rodada 
de testes mais ampla, envolvendo outros membros da equipe que não participaram diretamente do desenvolvimento, ajudando a ter uma visão da ferramenta sem este viés. A isso, seguiram-se novas correções e melhorias; 7) Release: Por fim, o plug-in foi disponibilizado para sua utilização dentro do AVA. Oportunidades de melhoria também surgiram com os feedbacks de usuários conforme foram utilizando a ferramenta, coletados por meio de questionários disponibilizados no AVA e também do monitoramento do uso da mesma.

\subsection{Linguagens de Desenvolvimento}

Visto que a ferramenta foi pensada para integrar a plataforma Moodle, como um módulo de atividade, ela foi implementada segundo os padrões de desenvolvimento correspondentes (Moodle, 2020a). A linguagem principal de desenvolvimento do sistema é PHP, uma linguagem de script de propósito geral (The PHP Group, 2020), associada a um banco de dados MySQL e servidor Apache, no sistema operacional Linux (Moodle, 2020b). Para o desenvolvimento do front-end, também são utilizadas as linguagens HTML, CSS e JavaScript. Por fim, foi necessário usar as seguintes bibliotecas adicionais: Google Maps JavaScript API e Google Charts, usadas na exibição de dados geográficos e gráficos, CKEditor, no recurso de edição de texto, e JqueryRate, para implementar um mecanismo de avaliação. Os requisitos mínimos de hardware indicados para a utilização da plataforma são um espaço em disco de 200MB, processador de $1 \mathrm{GHz}$ e memória de 512MB (Moodle, 2020b), mas tal infraestrutura precisa ser bem dimensionada, especialmente na perspectiva de adoção de cursos com uma quantidade expressiva de usuários.

\subsection{Características}

Para levantar que características do fórum que poderiam ser mais relevantes no estímulo a participação dos usuários, foi conduzido estudo de revisão sistemática da literatura (Mendes, Machiavelli e Gusmão, 2019a) em que foram pesquisadas referências prévias de utilização da Inteligência Artificial com esta finalidade, onde se concluiu que esta tecnologia não tem sido tão bem aproveitada quanto poderia. Tendo em vista o potencial de mecanismos de recomendação para aumentar o nível de interação dos usuários com os recursos do sistema, também decidiu-se realizar uma investigação na literatura de seu uso educacional (Mendes, Machiavelli e Gusmão, 2019b). Ambos os estudos, assim como o levantamento inicial realizado com a equipe acadêmica para entender os requisitos dos participantes, serviram para embasar o conjunto final de características a compor a ferramenta. Desta forma, quatro estratégias básicas foram definidas: 1) foco em aspectos visuais, de melhoria de usabilidade; 2) feedbacks aos cursistas por meio de notificações e aos gestores por meio de relatórios; 3) elementos de gamificação, que consiste em uma prática de incentivo à realização de determinadas atividades a partir de estratégias de design de jogos, tornando a experiência mais prazerosa (Massarolo e Mesquita, 2020); 4) mecanismo de recomendação de conteúdos e contatos, baseado em IA.

Além da sua função principal de envio de mensagens de texto, comum aos fóruns de discussão online, o plug-in desenvolvido tem as seguintes funcionalidades: Classificação do tipo de postagem - ao postar um determinado conteúdo, o usuário deve selecionar um dos tipos: (1) Pergunta, (2) Narrativa, e (3) Questão disparadora (esta, disponível apenas para moderadores do fórum, onde eles fazem publicações com destaque visual a fim de motivar a participação dos usuários nas discussões). A classificação permite uma melhor identificação da natureza da postagem; Tags - também com o objetivo de caracterizar a postagem, são rótulos que devem ser associados para indicar os principais assuntos abordados nas postagens; Curtidas e não curtidas - inspirando-se no funcionamento das redes sociais, os usuários têm a possibilidade de indicar se a postagem foi de seu agrado ou não, o que também serve como um feedback para o autor do conteúdo postado; Ranking - um dos recursos de gamificação, consiste em um painel que lista os usuários, por ordem de quantidade de interações. Este valor também pode ser contabilizado como uma pontuação extra para a nota do participante na atividade, caso isto seja configurado em sua criação. O objetivo deste recurso é estimular a colaboração e competição saudável entre os estudantes, e dar um reconhecimento público àqueles que se mostram mais engajados nas discussões; Chat - trata-se de um recurso que permite a comunicação síncrona dentro do próprio plug-in, não sendo necessário criar uma atividade acessória, a exemplo do que acontece no uso convencional do Moodle. É possível estabelecer relacionamentos por meio da adição de contatos, que podem ser provenientes de uma busca, ou sugeridos pelo mecanismo de recomendação, descrito posteriormente; Notificações - os participantes são informados de novas postagens ocorridas no fórum, e recomendações de pessoas e conteúdos personalizados, por meio de mensagens de e-mail; Relatórios - os cursos onde o plug-in é utilizado não contam com tutores, 
facilitadores ou professores, mas é preciso acompanhamento pela equipe de gestão, que deve ter uma visão geral do conteúdo discutido no fórum para entender melhor o perfil dos estudantes e suas necessidades de aprendizagem. Essas informações poderão subsidiar a disponibilização de novos conteúdos e materiais no próprio curso e embasar futuras ofertas educacionais. O relatório destaca quais são os temas mais populares e os usuários que são mais ativos, dados que também ajudam no diagnóstico do nível de participação do fórum. Os dados apresentados nos relatórios podem ser filtrados de acordo com a região geográfica, o assunto, a data, ou um usuário específico. Também é possível fazer associações entre essas informações e as postagens mais curtidas e não curtidas, ou comentários. Esta funcionalidade não está disponível para os estudantes, apenas para usuários com permissão de gerenciar os cursos; Recomendação de conteúdos e de contatos - nos cursos autoinstrucionais, abordados neste artigo, em que por definição não há um instrutor para orientar os estudantes sobre qual material consultar, o mecanismo de recomendação supre esta função, auxiliando os usuários na redução do espaço de busca de quais conteúdos a serem consumidos, considerando seus interesses e características nesta tomada de decisão. Isto é também um fator motivador, pois diminui a sobrecarga cognitiva de ter que avaliar inúmeras postagens em busca de algo relevante.

O mecanismo de recomendação foi implementado com base na técnica de IA de filtragem baseada em conteúdo, que considera apenas o histórico dos itens anteriormente acessados pelo usuário. A escolha desta abordagem se deu por sua maior facilidade de implementação, e menor custo computacional, restrições significativas dado o contexto do projeto. Os itens a serem recomendados podem ser: 1) Materiais e postagens: leva-se em consideração a associação desses itens a suas respectivas tags. Uma equação, cujos pesos são determinados pela quantidade de interações com uma dada tag, calcula o nível de interesse do usuário para o referido assunto; 2) Usuários: o sistema avalia o nível de expertise dos usuários em um determinado tema, ao identificar sua quantidade de postagens do tipo "Narrativa" criadas com a tag correspondente. Assim, caso um usuário seja definido como expert, ele pode ser recomendado como contato a outras pessoas que tenham interesse no assunto que ele demonstra dominar. Outros fatores levados em consideração neste cálculo são a quantidade de curtidas (que representam feedbacks positivos) e comentários. As não-curtidas, por sua vez, são contabilizadas negativamente, isto é, diminuem a reputação de expert de um usuário.

\section{CONCLUSÃO}

Até o momento da escrita desta publicação, a ferramenta estava sendo utilizada nos cursos autoinstrucionais promovidos pela Universidade Aberta do Sistema Único de Saúde da Universidade Federal de Pernambuco (UNA-SUS UFPE), a cargo do Grupo de Pesquisa SABER Tecnologias Educacionais e Sociais. O ambiente que disponibiliza estes cursos possui mais de 200.000 usuários, o que indica o amplo alcance da plataforma. Por serem cursos de formação continuada na área de Saúde, requerem boa interação dos participantes na troca de experiências profissionais, a fim de contemplar especificidades. O plug-in descrito tem sido um recurso valioso para o aprofundamento das discussões originadas a partir dos conteúdos dos cursos, capaz de atender necessidades individualizadas apresentadas pelos cursistas, a partir da indicação de recursos complementares ao aprendizado e pessoas que tenham interesse de discutir temas semelhantes. Este fato corrobora com a função do fórum de discussão de formar uma comunidade de aprendizado e reunir pessoas que possuam interesses comuns.

Outro aspecto que merece ser destacado é a facilidade que a equipe de gestão tem, com este recurso, de conhecer as demandas dos cursistas a partir do monitoramento frequente dos relatórios que o plug-in oferece. Com isso, é possível saber quais temas geram mais discussões e dúvidas e, quando necessário, favorece a disponibilização de conteúdos e materiais didáticos complementares que possam apoiar os estudantes no processo de aprendizado, considerando-se, inclusive, necessidades regionalizadas, algo muito comum na área da Saúde e que não é possível de se fazer com o fórum convencional do Moodle, pois carece de ferramentas de visualização e análise de dados desta natureza. Assim, é possível atender particularidades dos cursistas mesmo em experiências de aprendizagem massivas e melhorar a qualidade dos cursos durante a oferta dos mesmos.

Como trabalhos futuros, estão previstas melhorias tecnológicas, com destaque para a implementação de um mecanismo de recomendação mais refinado, incrementando a técnica de filtragem baseada em conteúdo para aumentar sua precisão, e estudos mais detalhados a fim de compreender os impactos desta ferramenta na aprendizagem. 


\section{AGRADECIMENTOS}

Este trabalho foi desenvolvido a partir de pesquisa financiada pelo CNPq (Conselho Nacional de Desenvolvimento Científico e Tecnológico), ao qual se agradece o apoio prestado.

\section{REFERÊNCIAS}

Alstete, J. e Beutell, N., 2004. Performance indicators in online distance learning courses: a study of management education. Quality Assurance in Education.

Bajaj, R. e Sharma, V., 2018. Smart Education with artificial intelligence based determination of learning styles. Procedia Computer Science, vol. 132, pp. 834-842.

Barros, D., Santos, V. e Romero, C., 2019. Estratégias para o trabalho colaborativo: revisitando o uso de fóruns on-line na educação a distância. Revista Diálogo Educacional, vol. 19, n. 60, pp. 221-245.

Bri, D.; García, M., Coll, H. e Lloret, J., 2009. A study of virtual learning environments. WSEAS Transactions on Advances in Engineering Education, vol. 6, n. 1, pp. 33-43.

Brown, D.; Ceretta, L. e Soratto, M., 2018. Utilização do Telessaúde na Educação Permanente pelos Enfermeiros da Estratégia da Saúde da Família. Enfermagem Brasil, vol. 17, n. 4, pp. 326-335.

Chassignol, M.; Khoroshavin, A.; Klimova, A.; Bilyatdinova, A. Artificial Intelligence trends in education: a narrative overview. Procedia Computer Science, vol. 136, pp.16-24.

Futterleib, J. e Santos, R., 2006. Ferramenta é instrumento, ambiente é cenário. E os fóruns on-line de discussão? Recortes de reflexão sobre o estudo da física em ambientes não-formais de educação. Revista de Ensino de Ciências $e$ Matemática, vol. 8, n. 1.

Herath, D. e Jayarathne, L., 2018. Intelligent Recommendations for e-Learning Personalization Based on Learner's Learning Activities and Performances. International Journal of Computer Science and Software Engineering, vol. 7, n. 6, jun. 2018, pp.130-137.

Kalay, Y., 2004. Virtual learning environments. Journal of Information Technology in Construction, vol. 9, n. 13, pp. 195-207.

Kanuka, H., 2005. An exploration into facilitating higher levels of learning in a text-based Internet learning environment using diverse instructional strategies. Journal of Computer Mediated Communication, vol. 10, n. 3.

Kolb, D., 1984. Experiential Learning: Experience as the Source of Learning and Development. Englewood Cliffs, NJ: Prentice-Hall.

Krämer, B. e Schulte D., 2008. The impact of technology on students at open and distance universities. In: F. Agrusti, D. Keegan, G.; Kismihok, B.; Krämer, N.; Mileva, D.; Schulte, J. e Thompson, B. Vertecchi. The impact of new technologies on distance learning students. Brussels: European Commission, pp. 28-48.

Krämer, B., 2002. Web-Based Learning Environments: Tools and Engineering Issues. In: The Handbook for Economics Lecturers: A Set of Guides to Inform and Improve the Practices of University Teachers of Economics. Bristol, UK: Learning and Teaching Support Network Generic Centre/ALT Guides, LTSN.

Lockwood, F. 1992. Activities in self-instructional texts. London: Kogan Page.

Loncar, M.; Barrett, N. e Liu, G., 2014. Towards the refinement of forum and asynchronous online discussion in educational contexts worldwide: Trends and investigative approaches within a dominant research paradigma. Computers \& Education, vol. 73, pp. 93-110.

Mason, R., 2011. Student Engagement with, and Participation in, an e-Forum. Educational Technology \& Society, vol. 14, n. 2, pp. 258-268.

Massarolo, J. e Mesquita, D., 2013. Narrativa transmídia e a Educação: panorama e perspectivas. Revista Ensino Superior Unicamp, vol. 9, p. 34-42.

McCarthy, J.; M. Minsky, N. Rochester, C. Shannon., 2006. A Proposal for the Dartmouth Summer Research Project on Artificial Intelligence. AI Magazine, vol. 27, n. 4.

McNamara, J e Brown, C., 2008. Assessment of collaborative learning in online discussions. ATN Assessment, vol. 1, n. 1.

Mendes, P. B; Machiavelli, J. L. e Gusmão, C. M. G. de, 2019a. Avaliação de Tecnologias de Recomendação de Conteúdo Baseadas em Inteligência Artificial Numa Visão Educacional. In: $25^{\circ}$ CIAED Congresso Internacional ABED de Educação a Distância, Poços de Caldas - MG.

Mendes, P. B; Machiavelli, J. L. e Gusmão, C. M. G. de, 2019b. Revisão Sistemática da Utilização de Fóruns Inteligentes para a Promoção da Colaboração na Aprendizagem Virtual. Revista Brasileira de Informática na Educação, vol. 27, n. 3 . 
Mengistu, D., 2016. The Relevance of Distance Education Print Instructional Materials to the Needs of Distance Learners: The Case of Ministry Of National Defense (Tese de Doutorado, Addis Ababa University).

Menon, M.; Bhat, V.; Mathew, R. e Phalachandra, B., 2016. A Study on the Adoption and Integration of OER Materials in Self-Instructional Course Development. Proceedings of Pan-Commonwealth Forum 8 (PCF8), 2016.

Mokoena, S., 2013. Engagement with and participation in online discussion forums. Turkish Online Journal of Educational Technology, vol. 12, n. 2, pp. 97-105.

Monahan, T.; Mcardle, G. e Bertolotto, M., 2008. Virtual reality for collaborative e-learning. Computers \& Education, vol. 50, n. 4, pp. 1339-1353.

Moodle, 2018. About Moodle. Disponível em: https://docs.moodle.org/38/en/About_Moodle (Acessado em 11 jun. 2020)

Moodle, 2020a. Activity Modules. Disponível em: https://docs.moodle.org/dev/Activity_modules (Acessado em 11 jun. 2020)

Moodle, 2020b. Installing Moodle. Disponível em: https://docs.moodle.org/38/en/Installing_Moodle (Acessado em 11 jun. 2020)

Nwana, H., 1990. Intelligent tutoring systems: an overview. Artificial Intelligence Review, vol. 4, n. 4, pp. 251-277.

O'Leary, R. e Ramsden, A., 2002. Virtual learning environments. In: The Handbook for Economics Lecturers: A Set of Guides to Inform and Improve the Practices of University Teachers of Economics. Bristol, UK: Learning and Teaching Support Network Generic Centre/ALT Guides, LTSN.

Premagowrie, S.; Vaani, R. e Ho, R., 2014. Online Forum: A Platform that Affects Students' Learning?. American International Journal of Social Science, vol. 3, n. 7, December.

Shaw, R., 2012. A study of the relationships among learning styles, participation types, and performance in programming language learning supported by online forums. Computers \& Education, vol. 58, n.1, pp. 111-120.

Silva, M., 2008. Cibercultura e educação: a comunicação na sala de aula presencial e online. Revista FAMECOS, Porto Alegre, n. 37.

The PHP Group, 2020. What is PHP? Disponível em: https://www.php.net/manual/en/intro-whatis.php (Acessado em 11 jun. 2020)

Thomas, M., 2002. Learning within incoherent structures: The space of online discussion forums. Journal of Computer Assisted Learning, vol. 18, n. 3, pp. $351-366$.

Webb, E; Jones, A.; Barker, P. e Van Schaik, P., 2004. Using e-learning dialogues in higher education. Innovations in Education and Teaching International, vol. 41, n.1, pp. 93 - 103. 